\title{
Filtro de partículas aplicado a estimativa do campo de temperaturas no interior de um cérebro sujeito a um tratamento com eletrodo DBS
}

\author{
Caroline R. Pereira ${ }^{1}$ \\ Luiz A. S. Abreu ${ }^{2}$ \\ Diego C. Knupp ${ }^{3}$ \\ IPRJ/UERJ, Nova Friburgo, RJ
}

\begin{abstract}
Resumo. Este trabalho trata da estimativa sequencial da temperatura interna do cérebro contendo um eletrodo DBS através da solução de um problema de estimativa de estado com o método de filtro de partículas. A equação clássica de biotransferência de calor bidimensional foi considerada na formulação matemática. O problema direto associado foi resolvido com uma abordagem de elementos finitos implementada por meio da função NDSolve, disponível no software Wolfram Mathematica ${ }^{\circledR}$. O problema de estimativa de estado foi resolvido com o filtro de partículas algoritmo amostragem por importância e reamostragem sequencial (SIR) supondo que medições estejam disponíveis no eletrodo DBS. As incertezas nos modelos de evolução e de observação foram assumidas como aditivas, gaussianas, não correlacionadas e com média zero. As soluções mostraram uma abordagem promissora para reduzir os riscos de lesões relacionadas com a técnica da estimulação cerebral profunda, especialmente por levarem em conta as incertezas relacionadas com as medições.
\end{abstract}

Palavras-chave. DBS, Biotransferência de Calor, Problemas Inversos, Filtro de Partículas

\section{Introdução}

A estimulação cerebral profunda (do inglês, Deep Brain Stimulation - DBS) é uma terapia estabelecida que consiste no envio de pulsos elétricos a áreas cerebrais específicas por meio de eletrodos implantados no interior do cérebro [3-6,16]. O procedimento é realizado com o objetivo de estimular o funcionamento do órgão e melhorar o desempenho e qualidade de vida de pessoas que possuem doenças neurológicas como a doença de parkinson, a epilepsia, a distonia, entre outras $[3,6,16]$. No entanto, apesar do uso do DBS ser amplamente difundido em todo o mundo e ter um procedimento cirúrgico bem tolerado, pouco se sabe sobre seus efeitos fisiológicos no cérebro. Algumas das lesões mais comuns estão relacionadas a possíveis queimaduras internas por acoplamento térmico com outros equipamentos, como a ressonância magnética [4-6]. Desta forma, mudanças de temperatura induzidas por DBS são de amplo interesse e precisam ser controladas para minimizar o risco de lesões. Essas variações vem sendo analisadas por meio de solução de problemas diretos considerando modelos matemáticos aproximados do cérebro $[2,4,5]$ ou ainda modelos mais realistas obtidos a partir de imagens por ressonância magnética [7].

Ademais, podem ser empregadas técnicas de solução de problemas inversos que levam em consideração medidas experimentais para estimar algumas propriedades físicas presentes no modelo

\footnotetext{
${ }^{1}$ rpereiracaroline@iprj.uerj.br

${ }^{2}$ luiz.abreu@iprj.uerj.br

${ }^{3}$ diegoknupp@iprj.uerj.br
} 
matemático DBS [6]. A utilização de problemas inversos para a estimativa de parâmetros e obtenção do campo de temperatura mostra-se bastante relevante, pois permite que sejam inseridas no problema incertezas associadas ao modelo matemático e as propriedades, além de levar em consideração as medidas experimentais. Neste contexto, a solução de problemas inversos utilizando filtros Bayesianos vem sendo utilizada em problemas de biotransferência de calor. Diversos trabalhos podem ser encontrados empregando estimativas de estado para obtenção do campo de temperatura em tratamentos de câncer por hipertermia em diferentes partes do corpo humano $[8,9,13]$ e em isquemia cerebral [11]. Segundo o conhecimento dos autores não há nenhuma publicação onde os filtros de partículas foram aplicados em problemas envolvendo DBS. Então, o objetivo deste estudo é mostrar a possibilidade de estimar sequencialmente o campo de temperaturas internas do cérebro contendo um eletrodo DBS por meio da solução de um problema de estimativa de estado.

\section{Formulação e Solução do Problema de Biotransferência de Calor com DBS}

O problema físico considerado neste trabalho envolve a biotransferência de calor no cérebro com um estimulador elétrico DBS implantado. Assumindo a hipótese de que o problema possui simetria, a solução reduz-se a resolver o caso ilustrado na Figura 1(a). Este plano tem limites que variam de $r=R_{1}$ a $r=R_{2}$ e de $z=0$ a $z=H$, onde $R_{1}$ é o raio do eletrodo, $R_{2}$ e $\mathrm{H}$ são, respectivamente, uma distância e uma altura suficientemente longas para não influenciar na solução da simulação computacional. Além disso, é considerado que existe um sensor, localizado no centro do eletrodo, utilizado para obter medidas experimentais de temperatura. O aumento de temperatura será descrito com o modelo proposto por Pennes $[2,4-7,14]$ :

$$
\begin{gathered}
\rho c_{p} \frac{\partial T}{\partial t}=\frac{1}{r} \frac{\partial}{\partial r}\left(k r \frac{\partial T}{\partial r}\right)+\frac{\partial}{\partial z}\left(k \frac{\partial T}{\partial z}\right)-\rho_{b} \omega_{b} c_{b}\left(T-T_{b}\right)+Q_{m}+Q_{e x t}(r, z) \\
\left.\frac{\partial T}{\partial r}\right|_{r=R_{1}}=0 \\
T(r, z, t)=T_{a}, \quad \text { em } r=R_{2}, z=0, z=H \text { e } t=0,
\end{gathered}
$$

sendo, para o tecido do cérebro, $\rho$ a massa específica, $c_{p}$ o calor específico, $k$ a condutividade térmica e $Q_{m}$ a geração metabólica de calor e, para o sangue, $\rho_{b}$ a massa específica, $\omega_{b}$ a perfusão sanguínea, $c_{b}$ o calor específico e $T_{b}$ a temperatura. $T_{a}$ é a temperatura inicial do cérebro quando não há aquecimento elétrico. O termo $Q_{\text {ext }}(r, z)$ representa o aquecimento imposto por ondas de radiofrequência através de eletrodos implantados no cérebro [2,4-7,9, 14]. Assim, pode-se definir:

$$
Q_{\text {ext }}(r, z)=\sigma|\nabla V|^{2},
$$

onde $\sigma$ é a condutividade elétrica, $\nabla V$ o campo elétrico e $V(r, z)$ o potencial elétrico, obtido resolvendo a seguinte equação de Laplace $[2-7,9,14]$ :

$$
\begin{gathered}
\frac{1}{r} \frac{\partial}{\partial r}\left(\sigma r \frac{\partial V}{\partial r}\right)+\frac{\partial}{\partial z}\left(\sigma \frac{\partial V}{\partial z}\right)=0 \\
\frac{\partial V}{\partial \boldsymbol{n}}=0, \quad \text { em } r=R_{2}, \quad z=0 \text { e } z=H \\
V(r, z)=f(z), \text { em } r=R_{1} .
\end{gathered}
$$

A função $f(z)$ será definida de acordo com o modelo de eletrodo utilizado. Para a análise de resultados foram estudados dois eletrodos Medtronic ${ }^{\circledR}$ DBS: modelo 3387 e modelo 3389. Esses 
modelos são eletrodos cilíndricos com 1,27 $\mathrm{mm}$ de diâmetro e, em cada um, há quatro eletrodos com 1,5 $\mathrm{mm}$ de comprimento. A diferença entre eles é o espaçamento dos eletrodos, com distâncias de 0, $5 \mathrm{~mm}$ no modelo 3389 e 1,5 $\mathrm{mm}$ no modelo 3387, conforme apresentado na Figura 1(b) [6,10].

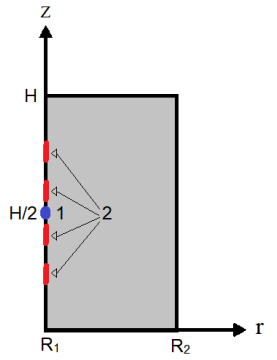

(a) Domínio axissimétrico onde 1 e 2 são as posições do sensor e dos eletrodos, respectivamente.

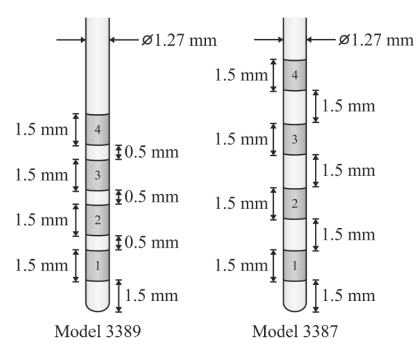

(b) Configuração geométrica dos eletrodos Fonte: Adaptado de [10].

Figura 1: Representação esquemática do domínio e dos eletrodos utilizados.

\section{Problemas de Estimativa de Estado}

Os problemas de estimativa de estado foram desenvolvidos para que os dados medidos disponíveis possam ser utilizados em conjunto com o conhecimento prévio sobre os fenômenos físicos, parâmetros geométricos e informações referentes aos dispositivos de medição, a fim de produzir sequencialmente estimativas das variáveis dinâmicas desejadas. Esses problemas podem ser escritos na forma de modelos de evolução de estado, equação (8), e observação, equação (9) $[9,11,12]$.

$$
\begin{gathered}
\boldsymbol{x}_{k}=\boldsymbol{f}_{k}\left(\boldsymbol{x}_{k-1}, \boldsymbol{\theta}, \boldsymbol{v}_{k}\right), \quad k=1, \ldots, M \\
\boldsymbol{z}_{k}=\boldsymbol{g}_{k}\left(\boldsymbol{x}_{k}, \boldsymbol{\theta}, \boldsymbol{n}_{k}\right), \quad k=1, \ldots, M,
\end{gathered}
$$

onde $\boldsymbol{x}_{k}$ é o vetor das variáveis de estado que descrevem o sistema em um instante de tempo $t_{k}, \boldsymbol{z}_{k}$ é a previsão relacionada as medições $\left(\boldsymbol{z}_{k}^{\text {meas }}\right), \boldsymbol{\theta}$ é o vetor de parâmetros do problema e $\boldsymbol{v}_{k}$ e $\boldsymbol{n}_{k}$ são os ruídos no modelo de evolução e observação, respectivamente. O objetivo do problema de estimativa de estado é obter informações sobre o vetor de estado $\boldsymbol{x}_{k}$ com base nos modelos de evolução e observação definidos pelas equações (8) e (9), considerando uma densidade de probabilidade $\pi\left(\boldsymbol{x}_{0}, \boldsymbol{\theta} \mid \boldsymbol{z}_{0}\right)=\pi\left(\boldsymbol{x}_{0}, \boldsymbol{\theta}\right)$ conhecida no tempo inicial $t_{0}[9,11,12]$.

Essas estimativas podem ser feitas com filtros Bayesianos que são métodos probabilísticos que utilizam um algoritmo recursivo para a estimativa e atualização dos estados. Existem vários tipos de filtros Bayesianos, para esse problema foi escolhido o filtro de partícula por se tratar de uma técnica poderosa e robusta que pode ser aplicada também a problemas não lineares [9, 11]. Neste caso, o filtro de partículas escolhido foi o algoritmo amostragem por importância e reamostragem sequencial (do inglês, Sampling Importance Resampling- SIR), descrito na Tabela 1 [9, 11, 12]. Os modelos de evolução serão dado pelas soluções das equações (1) a (7). Além disso, as medições são assumidas aqui como não correlacionadas e contendo ruídos gaussianos aditivos, com médias zero e uma matriz de covariância constante $\boldsymbol{R}$. Com tais hipóteses sobre os erros de medição, a função de verossimilhança, usada para o cálculo dos pesos no método do filtro de partículas, é dada por [11]:

$$
\pi\left(\mathbf{z}_{k} \mid \mathbf{x}_{k}, \boldsymbol{\theta}\right)=(2 \pi)^{-I / 2}|\boldsymbol{R}|^{-1 / 2} \exp \left\{-\frac{1}{2}\left[\boldsymbol{z}_{k}^{\text {meas }}-\boldsymbol{g}_{k}\left(\boldsymbol{x}_{k}, \boldsymbol{\theta}\right)\right]^{T} \boldsymbol{R}^{-1}\left[\boldsymbol{z}_{k}^{\text {meas }}-\boldsymbol{g}_{k}\left(\boldsymbol{x}_{k}, \boldsymbol{\theta}\right)\right]\right\},
$$


onde $I$ é o número de medições em cada instante $t_{k}$. O vetor de parâmetros do modelo é assumido como conhecido, mas as incertezas relacionadas são contabilizadas nos vetores de ruído $v_{k}$ e $n_{k}$.

Tabela 1: Algoritmo amostragem por importância e reamostragem sequencial (SIR).

\begin{tabular}{|c|}
\hline Passo 1 \\
\hline $\begin{array}{l}\text { Para } i=1, \ldots N \text {, onde } N \text { é o número de partículas, gere um conjunto de partículas da } \\
\text { distribuição inicial } \mathbf{x}_{k}^{i}=\pi\left(\mathbf{x}_{k} \mid \mathbf{x}_{k-1}^{i}, \boldsymbol{\theta}\right) \text { e calcule os pesos } \omega_{k}^{i}=\pi\left(\mathbf{z}_{k} \mid \mathbf{x}_{k}^{i}, \boldsymbol{\theta}\right)\end{array}$ \\
\hline Passo 2 \\
\hline Calcule o peso total $T_{\omega}=\sum_{i=1}^{N} \omega_{k}^{i}$ e normalize os pesos $\widehat{\omega}_{k}^{i}=\frac{\omega_{k}^{i}}{T_{\omega}}$. \\
\hline Passo 3 \\
\hline $\begin{array}{l}\text { Reamostragem das partículas: } \\
\text { Construa a soma dos pesos acumulativos (do inglês, cumulative sum of weights - CSW), } \\
\text { sendo computada por } c_{i}=c_{i-1}+\widehat{\omega}_{k}^{i} \text { com } c_{0}=0 \text {. } \\
\text { Tome } i=1 \text { e gere } u_{1} \text { de uma distribuição uniforme } U\left[0, N^{-1}\right] . \\
\text { Para } j=1,2, \ldots, N \text {, calcule } u_{j}=u_{1}+N^{-1}(j-1) \text { e enquanto } u_{j}>c_{i} \text {, faça } i=i+1 ; \\
\text { Designe as partículas } \mathbf{x}_{k}^{j}=\mathbf{x}_{k}^{i} \text { e os pesos para } \omega_{k}^{j}=N^{-1} \text {. }\end{array}$ \\
\hline
\end{tabular}

\section{Resultados e Discussões}

Neste trabalho foi utilizado o NDSolve do software Wolfram Mathematica ${ }^{\circledR}$ para a solução do problema direto, escolhendo como método de solução o Método dos Elementos Finitos (MEF) com uma malha composta por 3034 elementos triangulares. Os parâmetros do problema, listados na Tabela 2, foram considerados constantes e podem ser encontrados na literatura [5,6]. A temperatura inicial será $T_{a}=37^{\circ} \mathrm{C}$. A condição proposta na equação (7) deve ser definida como uma função escalonada. Neste caso, $f(z)=V_{r m s}$ nas posições dos eletrodos ativos e $f(z)=0$ nas posições inativas, sendo $V_{r m s}=1,561 \mathrm{~V}$, obtido a partir de um sinal elétrico de $10 \mathrm{~V}, 185$ pps e $210 \mathrm{~ms}[5,6]$. A solução é apresentada nas Figuras 2(a) e 2(b), que representam a distribuição do potencial elétrico e da temperatura, considerando o tempo de 60 segundos. O par de eletrodos ativos selecionados é o 1 e 4 da Figura 1(b) para o modelo 3389.

Após a solução do problema direto, 60 medidas de temperatura experimentais simuladas foram obtidas a cada $1 s$ na posição do sensor da Figura 1(b), utilizando uma distribuição normal com média zero e desvio padrão de $0,05^{\circ} \mathrm{C}$. A partir dessas temperaturas, os problemas de estimativa de estado foram resolvidos usando um único ponto de medição dentro do domínio. Inicialmente, como estes são resultados preliminares, a escolha das incertezas nos modelos de evolução e de observação foram, por simplicidade, arbitrariamente assumidas como aditivas, gaussianas, não correlacionadas e com média zero, como usualmente se encontra na literatura relacionada com biotransferência de calor $[8,9,11,13]$. Espera-se em trabalhos futuros analisar com mais detalhes sobre os erros de modelo associados com o modelo de observação e de evolução e, com isso, ter uma resposta mais detalhada sobre o impacto desta escolha $[1,15]$. Ademais, o termo fonte de calor resultantes do aquecimento foi considerado com ruído gaussiano aditivo e seu modelo de evolução é dado por [9]:

$$
Q_{\text {ext }, k}^{i}(r, z)=Q_{e x t, k-1}^{i}(r, z)+\epsilon_{k}^{i}(r, z),
$$

$\epsilon_{k}^{i}(r, z)$ tem média zero e desvio padrão de $1 \%$ de $Q_{e x t, k-1}^{i}(r, z)$. Para verificar o efeito do número de partículas utilizado no algoritmo SIR aplicou-se o erro quadrático médio [9,11], a saber, 


$$
R M S=\frac{\sqrt{\sum_{p=1}^{P}\left(T_{e s t, p}-T_{e x a, p}\right)^{2}}}{P},
$$

onde $T_{\text {est,p }}$ e $T_{\text {exa,p }}$ denotam, respectivamente, as temperaturas estimada e exata obtida com o sensor, e $P=61$ o número total de etapas de tempo onde as temperaturas foram comparadas. Na Tabela 3 encontram-se os resultados para $N=100, N=250$ e $N=500$ partículas com os eletrodos 1 e 4 ativos. Os erros RMS são reportados em termos de suas médias e valores de desvio padrão, obtidos com 10 execuções de cada algoritmo, para evitar qualquer viés resultante das medidas simuladas. Os tempos computacionais referem-se a uma única rodada dos códigos executados em um processador Intel(R) Core(TM) i7-8550U@1.80GHz com 8 GB de memória RAM.

Observando a Tabela 3 é possível verificar que ao aumentar o número de partículas o tempo de CPU aumenta e o erro RMS não apresenta muitas variações. Escolhendo $N=250$ foi obtido o campo de temperatura estimado em $t=60 \mathrm{~s}$, Figura 2(c), considerando o modelo $3389 \mathrm{com}$ os eletrodos 1 e 4 ativos. Nas Figuras 3(a)-3(d) encontram-se os perfis de temperatura ao longo do tempo para os dois modelos com diferentes pares de eletrodos ativos, onde observa-se que o campo de temperatura pode ser estimado com precisão em ambos os eletrodos e com diferentes posições ativas, apesar das incertezas nos modelos de evolução e observação e nas medições.

Tabela 2: Valores dos parâmetros.

\begin{tabular}{cccccccc}
\hline Parâmetro & Valor & Parâmetro & Valor & Parâmetro & Valor & Parâmetro & Valor \\
\hline$T_{b}\left({ }^{\circ} \mathrm{C}\right)$ & 36,7 & $R_{1}(\mathrm{~mm})$ & 0,635 & $\rho_{b}\left(\mathrm{~kg} / \mathrm{m}^{3}\right)$ & 1057 & $Q_{m}\left(\mathrm{~W} / \mathrm{m}^{3}\right)$ & 9132 \\
$\rho\left(\mathrm{kg} / \mathrm{m}^{3}\right)$ & 1040 & $R_{2}(\mathrm{~mm})$ & 20,0 & $c_{b}\left(\mathrm{~J} / \mathrm{kgK}^{3}\right)$ & 3600 & $\sigma(\mathrm{S} / \mathrm{m})$ & 0,35 \\
$c_{p}(\mathrm{~J} / \mathrm{kgK})$ & 3650 & $H(\mathrm{~mm})$ & 50,0 & $\omega_{b}\left(\mathrm{ml} / \mathrm{scm}^{3}\right)$ & 0,008 & $k(\mathrm{~W} / \mathrm{mK})$ & 0,527 \\
\hline
\end{tabular}

Tabela 3: Erros RMS e tempo de CPU.

\begin{tabular}{cccc}
\hline Número de Partículas & Erro RMS médio & Desvio padrão do erro RMS & Tempo de CPU \\
\hline 100 & $2,07 \times 10^{-3}$ & $7,66 \times 10^{-5}$ & $7,61 \mathrm{~min}$ \\
250 & $2,03 \times 10^{-3}$ & $2,72 \times 10^{-5}$ & $12,9 \mathrm{~min}$ \\
500 & $2,00 \times 10^{-3}$ & $3,05 \times 10^{-5}$ & $24,2 \mathrm{~min}$ \\
\hline
\end{tabular}

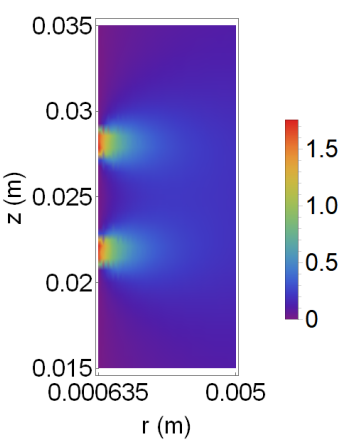

(a) Potencial elétrico $(V)$.

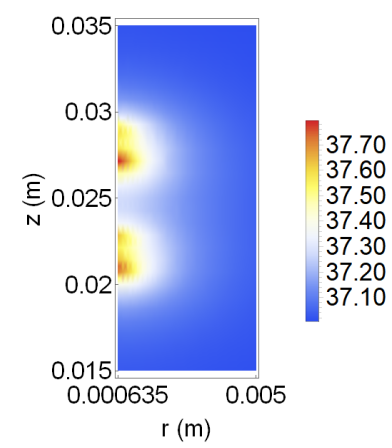

(b) Temperatura exata $\left({ }^{\circ} \mathrm{C}\right)$.

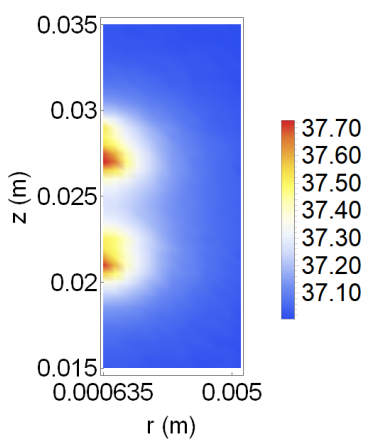

(c) Temperatura estimada $\left({ }^{\circ} \mathrm{C}\right)$.

Figura 2: Perfis de temperatura e potencial elétrico para o modelo $3389 \mathrm{em} t=60 \mathrm{~s}$. 


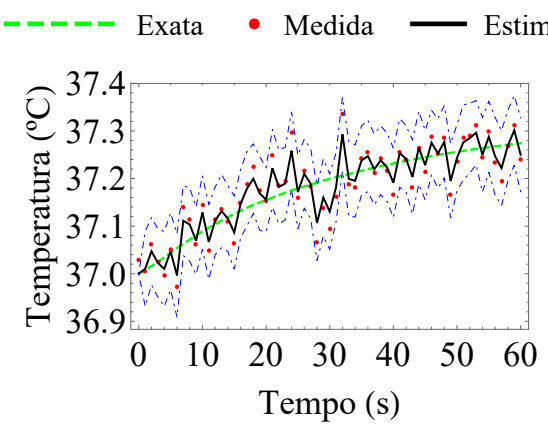

(a) Modelo 3389 e eletrodos 1 e 4 ativos.

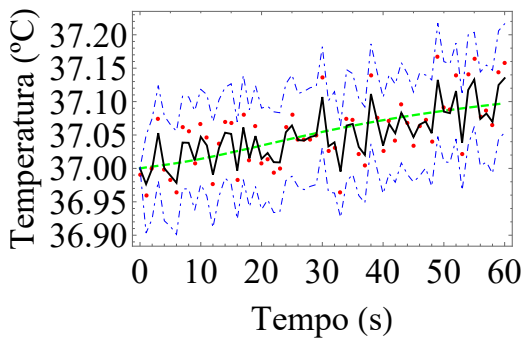

(c) Modelo 3387 e eletrodos 1 e 4 ativos.

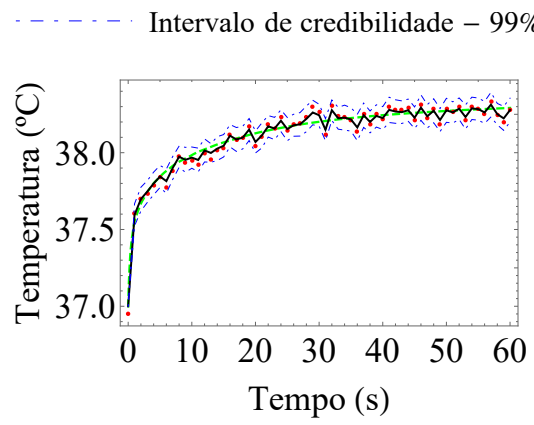

(b) Modelo 3389 e eletrodos 2 e 3 ativos.

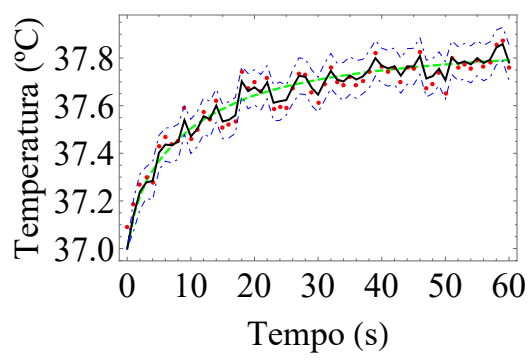

(d) Modelo 3387 e eletrodos 2 e 3 ativos.

Figura 3: Temperatura exata, estimada e medida na posição de localização do sensor.

\section{Conclusões}

Este artigo tratou da solução de problemas de estimativa de estado relacionados a atividade elétrica do DBS no cérebro. Com o objetivo melhorar a precisão do campo de temperaturas estimadas levando em consideração o maior número de incertezas possível. O algoritmo SIR, que leva em consideração as incertezas nos modelos de evolução e observação e nas medições, foi utilizado. Os resultados mostram que as temperaturas estimadas estavam em excelente acordo com as temperaturas exatas para os dois eletrodos DBS examinados considerando diferentes combinações de eletrodos ativos. Portanto, as soluções apresentadas mostraram uma abordagem promissora para reduzir os riscos de lesões relacionadas com a técnica da estimulação cerebral profunda.

\section{Agradecimentos}

O presente trabalho foi realizado com apoio da Coordenação de Aperfeiçoamento de Pessoal de Nível Superior - Brasil (CAPES)- Código de Financiamento 001, do Conselho Nacional de Desenvolvimento Científico e Tecnológico ( $\mathrm{CNPq}$ ) e da Fundação Carlos Chagas Filho de Amparo à Pesquisa do Estado do Rio de Janeiro (FAPERJ).

\section{Referências}

[1] Castello, D.A. e Ritto, T.G. Quantificação de incertezas e estimação de parâmetros em dinâmica estrutural: uma introdução a partir de exemplos computacionais. Notas em matemática aplicada, volume 81, 2016. 
[2] Chang, I. Finite element analysis of hepatic radiofrequency ablation probes using temperaturedependent electrical conductivity, Biomedical engineering online, 2:1-18, 2003. DOI: $10.1186 / 1475-925 \mathrm{X}-2-12$.

[3] Cubo, R. e Medvedev, A. Online Tissue Conductivity Estimation in Deep Brain Stimulation, IEEE Transactions on Control Systems Technology, 28:149-162, 2018. DOI: 10.1109/TCST.2018.2862397.

[4] Elwassif, M. M., Datta, A., Rahman, A. e Bikson, M. Temperature control at dbs electrodes using a heat sink: experimentally validated fem model of dbs lead architecture, Journal ofNeural Engineering, 9:1-9, 2012. DOI: 10.1088/1741-2560/9/4/046009.

[5] Elwassif, M. M., Kong, Q. Varquez, M. e Bikson, M. Bio-heat transfer model of deep brain stimulation-induced temperature changes, Journal ofNeural Engineering, 3:306-315, 2006. DOI: 10.1109/IEMBS.2006.259425.

[6] Jardim, L. C. S., Abreu, L. A. S., Knupp, D. C. e Silva Neto, A. J. Brain Thermal and Electrical Properties Estimation Using Experimental Data From Deep Brain Stimulation Lead, Revista Mundi Engenharia, Tecnologia e Gestão, 5:291-01,291-15, 2020. DOI: $10.21575 / 25254782 \mathrm{rmetg} 2020 \mathrm{vol} 5 \mathrm{n} 61349$.

[7] Khadka, N., Harmsen, I.E., Lozano, A.M. e Bikson, M. Bio-heat model of kilohertz-frequency deep brain stimulation increases brain tissue temperature, Neuromodulation: Technology at the Neural Interface, 23:489-495, 2020. DOI: 10.1111/ner.13120.

[8] Lamien, B., Orlande, H.R.B. e Eliçabe, G.E. Particle Filter and Approximation Error Model for State Estimation in Hyperthermia, Journal of Heat Transfer, 139:012001-3, 2017. DOI:10.1115/1.4034064

[9] Lamien, B., Varon, L.A.B. e Orlande, H.R.B. State estimation in bioheat transfer: a comparison of particle filter algorithms, International Jornal of Numerical Methods for Heat \& Fluid Flow, 27:615-638, 2016. DOI: 10.1108/HFF-03-2016-0118.

[10] Medtronic; INC. DBS Lead Kit for Deep Brain Stimulation, Model 3387/3389, Implant Manual. Minneapolis, MN, US: [s.n.], 2002.

[11] Nunes, F. S., Orlande, H. R. B. e Nowak, A. J. Estimation of the ischemic brain temperature with the particle filter method, Computer Assisted Methods in Engineering and Science, 26:519, 2019. DOI: $10.24423 /$ cames.252.

[12] Orlande, H.R.B., Dulikravich, G.S. e Colaço, M.J. Application of Bayesian Filters to Heat Conduction Problems, International Conference on Engineering Optimization, 1:1-5, 2008.

[13] Pacheco, C., Orlande, H.R.B., Colaco, M. e Dulikravich, G.S. State estimation problems in PRF-shift magnetic resonance thermometry, International Journal of Numerical Methods for Heat \& Fluid Flow, 28:315-335, 2018. DOI: 10.1108/hff-10-2016-0427

[14] Pennes, H. H. Analysis of tissue arterial blood temperature in the resting forearm, Journal of Applied Physiology, 1:93-122, 1948. DOI: 10.1152/jappl.1948.1.2.93.

[15] Soize, C. Stochastic models of uncertainties in computational mechanics. ASCE Society of Civil Engineers (Lecture notes in mechanics 2), 2012.

[16] Tierney, T. S. Deep brain stimulation foundations and future trends, Frontiers in Bioscience, 23:162-182, 2018. DOI:10.2741/4586 